OPEN ACCESS

Edited by:

Jie Yin,

Institute of Subtropical Agriculture

(CAS), China

Reviewed by:

Atte Von Wright

University of Eastern Finland, Finland

Ana Griselda Binetti,

Instituto de Lactología Industrial

(INLAIN, CONICET), Argentina

Jinping Chen,

Guangdong Academy of Sciences

(CAS), China

*Correspondence: Gang Wang

wanggang@jiangnan.edu.cn

Specialty section:

This article was submitted to

Food Microbiology,

a section of the journal

Frontiers in Microbiology

Received: 01 April 2019

Accepted: 12 July 2019

Published: 13 August 2019

Citation:

Wang L, Chen C, Cui S, Lee Y-k, Wang $\mathrm{G}$, Zhao J, Zhang $\mathrm{H}$ and

Chen W (2019) Adhesive

Bifidobacterium Induced Changes in Cecal Microbiome Alleviated

Constipation in Mice.

Front. Microbiol. 10:1721

doi: 10.3389/fmicb.2019.01721

\section{Adhesive Bifidobacterium Induced Changes in Cecal Microbiome Alleviated Constipation in Mice}

\author{
Linlin Wang ${ }^{1,2,3}$, Cailing Chen 1,2, Shumao Cui 1,2,3,4, Yuan-kun Lee ${ }^{5}$, Gang Wang ${ }^{1,2,3,4 *}$, \\ Jianxin Zhao ${ }^{1,2,4}$, Hao Zhang ${ }^{1,2,4,6,7}$ and Wei Chen ${ }^{1,2,6,8}$

\begin{abstract}
${ }^{1}$ State Key Laboratory of Food Science and Technology, Jiangnan University, Wuxi, China, ${ }^{2}$ School of Food Science and Technology, Jiangnan University, Wuxi, China, ${ }^{3}$ International Joint Research Laboratory for Probiotics, Jiangnan University, Wuxi, China, ${ }^{4}$ Institute of Food Biotechnology, Jiangnan University, Yangzhou, China, ${ }^{5}$ Department of Microbiology and Immunology, National University of Singapore, Singapore, Singapore, ${ }^{6}$ National Engineering Research Center for Functional Food, Jiangnan University, Wuxi, China, ${ }^{7}$ Wuxi Translational Medicine Research Center and Jiangsu Translational Medicine Research Institute Wuxi Branch, Wuxi, China, ${ }^{8}$ Beijing Advanced Innovation Center for Food Nutrition
\end{abstract} \\ and Human Health, Beijing Technology and Business University, Beijing, China
}

Constipation, which seriously affects living quality of people, is a common gastrointestinal disease. The engagement of the intestinal flora in the development of symptoms of constipation has been frequently hypothesized. In this study, constipated mice induced by loperamide were used to investige the alleviation of constipation by Bifidobacteria. Bifidobacteria was sorted out according to their adhesive properties into two groups. One group combined multiple strains of Bifidobacterium with adhesion property (CMB1), the other combined multiple strains of Bifidobacterium without adhesion property (CMB2). It was found that CMB1 can alleviate constipation more efficiently by improving the water, propionate and butyrate content in feces, and overall gastrointestinal transit time. Meanwhile, from the perspective of fecal microbiota, CMB1 alleviated constipation mainly by increasing the relative abundances of genera (Bifidobacterium, Lactobacillus, and Prevotella) associated with rapid bowel movement. From the perspective of cecal microbiota, CMB1 alleviated constipation mainly by increasing the relative abundances of genera Lactobacillus, Bacteroides, unclassified S24-7, Dorea, Ruminococcus, Coprococcus, and Rikenella, and decreasing the relative abundances of genera Oscillospira, Odoribacter and Unclassified F16, which are associated with methane production and colonic transit. Overall, changes of microbiota in caecum by CMB1 reflect the stage of constipation in mice more comprehensively than that in feces.

Keywords: Bifidobacterium, constipation, adhesion properties, SCFAs, gut microbiota

\section{INTRODUCTION}

Usually, constipation is defined as infrequent or hard to pass bowel movements. It has a high incidence (2-36\%) worldwide and complicated etiology (Rao et al., 2016). Its clinical symptoms include hard and dry feces, bowel movements that occur less than three times per week, rare occurrence of loose stools in the absence of laxatives and inadequate criteria for irritable bowel 
syndrome (Bharucha et al., 2013). In the past few years, dietary changes and the effects of physiological, psychological, sociological and other factors have led to an increase in the prevalence of constipation, which can severely affect people's health and quality of life. Although the harmfulness of constipation is limited, it can be linked to increased risk of many related diseases such as Parkinson disease (Rossi et al., 2015) and colorectal cancer (Guérin et al., 2014). Therefore, it is very necessary to prevent and treat constipation. At present, the main drugs used to treat constipation are osmotic and secretory laxatives (Zhang et al., 2015). However, these therapies are susceptible to drug resistance to varying degrees and some lack efficacy. Many studies have shown that gut flora played a vital role in constipation. Constipation is related to the changes of gut microbiota, which may disturb cross-talk between the intestinal microflora, the intestinal endocrine system, the immune system and intestinal permeability (Caesar et al., 2015; Ge et al., 2017). Recent studies have revealed that constipation is related to imbalance in the intestinal microbiota, which mainly involve reduced levels of Bifidobacterium or Lactobacillus and an increase in pathogens (Chen et al., 2016). Supplementation with Bifidobacteria (Peter et al., 2006; Urita et al., 2015) or lactobacilli (Drouault et al., 2008; Williams et al., 2009; Yoon et al., 2015), either alone or combined, could prevent or treat constipation $(\mathrm{Bu}$ et al., 2007; Tabbers et al., 2011).

At present, many studies about the relationship between intestinal flora and diseases were based on fecal samples, which are easy to obtain but do not fully reflect the intestinal microbiota. In mice, microbiota in the caecum ferment carbohydrates that are unavailable in the small intestine. And it has been reported that there are significant differences in the structure of fecal, caecal and mucous membranes microbiota (Mao et al., 2016). Therefore, it is necessary to study the relationship between caecum content microbiota and constipation. In addition, our previous studies indicated that alleviation of constipation by Bifidobacterium might be related to the adhesion property of the strains (Wang et al., 2017a). It thus suggested that adhesion property may facilitate colonization of Bifidobacterium in the intestine. To date, there has been no reported research on the effect of adhesion property of Bifidobacterium on constipation. Therefore, it is of great significance to study the difference between fecal microbiota and caecum microbiota in mice after treated with Bifidobacteria with or without adhesion property.

In this study, constipated mice induced by loperamide were used to investigate the alleviation of constipation by Bifidobacteria. The Bifidobacteria were sorted by their adhesive property. It was found that strains with adhesion property can alleviate constipation more efficiently.

\section{MATERIALS AND METHODS}

\section{Reagents}

Kits used to measure the levels of gastrointestinal (GI) neurotransmitters, motilin (MTL), gastrin (Gas), substance $\mathrm{P}$ (SP), endothelin (ET-1), somatostatin (SS) and vasoactive intestinal peptide (VIP) were purchased from Wen LE Bioengineering Institute (Shanghai, China).

Loperamide was dissolved in sterile water and its ultimate density was $1 \mathrm{mg} / \mathrm{mL}$. For acticarbon solution, gum arabic $100 \mathrm{~g}$ and water $800 \mathrm{~mL}$ were boiled until the solution was transparent. Acticarbon $50 \mathrm{~g}$ was then added and boiled three times. After cooling, the solution was diluted with water to $1000 \mathrm{~mL}$ and stored at $4^{\circ} \mathrm{C}$. Shake well before use.

\section{Bacteria Preparation}

Ten Bifidobacterium strains from five species obtained from American Type Culture Collection (ATCC) or China General Microbiological Culture Collection (CGMCC) were stored at the Culture Collection of Food Microorganisms in Jiangnan University (CCFM, Wuxi, Jiangsu Province, China). All the strains were cultured under anaerobic conditions for 24-48 h at $37^{\circ} \mathrm{C}$ in modified MRS (cMRS) broth supplemented with $0.05 \% \mathrm{w} / \mathrm{v} \mathrm{L}$-cysteine-HCl (Merck). To prepare active cultures for all experiments, all strains were consecutively reactivated in an anaerobic atmosphere at least three times using 3\% (v/v) inoculum in cMRS broth at $37^{\circ} \mathrm{C}$ for $24-48 \mathrm{~h}$ before use. To use these strains in the animal experiments, the bacterial culture was centrifuged at $5000 \times g$ for $10 \mathrm{~min}$, washed twice with PBS, and centrifuged again to obtain the bacteria. The bacteria were divided into two groups (CMB1 and CMB2) of five based on their adhesion properties (Table 1). Adhesion properties of all these bacteria had been checked by cell adhesion assay in vitro and their adhesion characteristics can be found in our previous studies (Wang et al., 2017a). CMB1 refers to the multi-Bifidobacteria combination with the final concentration of $10^{10} \mathrm{CFU} / \mathrm{mL}$ by mixing all Bifidobacteria with adhesion properties at the same concentration. The preparation method of CMB2 was the same as that of CMB1.

\section{Experimental Design}

Eight-week-old male BALB/c mice were obtained from Shanghai Laboratory Animal Center (Shanghai, China). The mice were kept in polyvinyl chloride (PVC) cages under environmentally controlled conditions with a 12-h light-dark cycle and standard commercial mouse feed and water were provided ad libitum. This study was approved by the Ethics Committee of the Jiangnan University, China (JN. No. 20150326-0110-21) and performed at the Experimental Animal Center of the Jiangnan University [License No. SYXK(SU)2016-0045].

To examine the preventive effects of $\mathrm{CMB}$ on constipation, 32 mice were used after a week-long adaptive period. The mice were randomly separated into four groups $(n=8)$ : normal (healthy mice), control (constipation mice without treatment), CMB1 (constipation mice treated with $\mathrm{CMB}$ with adhesion properties) and CMB2 (constipation mice treated with $\mathrm{CMB}$ with no adhesion properties).

The mice were fasted overnight before the first experiment. The normal and control groups were given $0.25 \mathrm{~mL}$ normal saline (NS) using intragastric administration once a day for 17 days. The CMB1 and CMB2 groups were intragastrically administered $0.25 \mathrm{~mL}$ of normal saline solution containing $4 \times 10^{10} \mathrm{CFU} / \mathrm{mL} \mathrm{CMB} 1$ or $\mathrm{CMB} 2$, respectively, daily for 
TABLE 1 | Bifidobacteria used in this study.

\begin{tabular}{|c|c|c|c|c|}
\hline Group & Strains & Source & Ways to acquire & Characteristics \\
\hline \multirow[t]{5}{*}{ CMB1 } & Bifidobacterium longum CCFM 643 & Human feces & Acquired CGMCC1.3048 & All strains had adherence property. \\
\hline & Bifidobacterium breve CCFM 670 & Infant feces & Acquired CGMCC1.3013 & \\
\hline & Bifidobacterium bifidum CCFM 16 & Infant feces & Acquired CGMCC1.3001 & \\
\hline & Bifidobacterium adolescentis CCFM 669 & Human feces & Acquired ATCC15701 & \\
\hline & Bifidobacterium animalis CCFM 625 & Mixed fermentation bacteria & Acquired ATCC15703 & \\
\hline \multirow[t]{5}{*}{ CMB2 } & Bifidobacterium longum CCFM 642 & Infant feces & Acquired ATCC15705 & All strains had no adherence property. \\
\hline & Bifidobacterium breve CCFM 622 & Infant feces & Screened & \\
\hline & Bifidobacterium bifidum CCFM 641 & Infant feces & Acquired ATCC29521 & \\
\hline & Bifidobacterium adolescentis CCFM 626 & Health human intestinal flora & Acquired CGMCC1.3003 & \\
\hline & Bifidobacterium animalis CCFM 624 & Rabbit feces & Acquired CGMCC1.1852 & \\
\hline
\end{tabular}

CMB1, combining multiple strains of Bifidobacterium with adhesion property; CMB2, combining multiple strains of Bifidobacterium with no adherence property. CGMCC, China General Microbiological Culture Collection; ATCC, American Type Culture Collection.

2 weeks. All of the groups, except the normal group, were given loperamide $(0.25 \mathrm{~mL})$ intragastrically from day 15 to day 17 to induce constipation (Qian et al., 2013). Changes in food intake, water intake, and body weight were measured once per day at 9:00 AM throughout the experimental period. On the last day of the experiment, the mice were anesthetized by dipping ether with cotton in a relatively sealed space. The mouse beard was cut with surgical scissors to prevent hemolysis. Fixed the mouse, used the tweezers to clamp the eyeball, let the blood flow vertically into the centrifuge tube, put the centrifuge tube at room temperature for $2 \mathrm{~h}$ and then put it into the $4^{\circ} \mathrm{C}$ refrigerator for $3 \mathrm{~h}$ and centrifuged at $3000 \times g$ for $15 \mathrm{~min}$ to obtain serum. The abdomen was opened, the cecum of the mouse was cut with a surgical scissors and the contents of the cecum were aspirated into the EP tube using a syringe without a needle, then gently put the colon in sterile saline to remove the remaining contents, and finally placed the colon into the EP tube and stored at $-80^{\circ} \mathrm{C}$ until analysis. All operations were performed under sterile conditions. The experimental design is shown in Supplementary Figure S1.

\section{Detection of Constipation-Related Indices}

The relative indices of constipation (i.e., the water content of the feces, the small intestinal transit rate and the first black stool defecation time), stool collection and SCFAs in feces were measured as previously described (Wang et al., 2017a). Feces were collected every three days and stored at $-80^{\circ} \mathrm{C}$. The water content of the feces was measured by the difference between the wet and dry weights of the feces, the small intestinal transit rate was evaluated by the distance traveled by an acticarbon solution relative to the overall length of the small intestine and the first black stool defecation time was measured by the time between the gavage of acticarbon solution and the appearance of darkened feces.

\section{Detection of the GI Neurotransmitters Levels in Serum}

The GI neurotransmitters levels in the serum were determined by an enzyme-linked immunosorbent assay (ELISA) instrument according to the manufacturer's instructions (Microplate Spectrophotometer Multiskan Go, Thermo Scientific, Waltham, MA, United States). The experiments mainly include the preparation of standard curve and the detection of sample absorption value (OD450) according to the standard curve in units of $\mathrm{ng} / \mathrm{L}$.

\section{$16 S$ rDNA Sequencing and Bioinformatics Analysis}

The enteric microorganisms in the fecal and caecal samples were measured using a metagenomics method (Wang et al., 2017a). Microbial genomic DNA was obtained using a FastDNA Spin Kit for Soil (MP Biomedical). The V4 region of the 16S rDNA was amplified by PCR. The products were purified and quantified using Gene Clean Turbo (MP Biomedical) and the Quant-iT PicoGreen dsDNA Assay Kit (Life Technologies), respectively. Libraries were prepared using TruSeq DNA LT Sample Preparation Kits (Illumina) and sequenced by Illumina MiSeq using the MiSeq Reagent Kit.

The QIIME pipeline was used to analyze the $16 \mathrm{~S}$ rDNA sequence data (Caporaso et al., 2010). The raw sequences were screened. The short lengths $(<200 \mathrm{bp})$ were then removed, and the pair-end reads that overlapped longer than $10 \mathrm{bp}$ and without any mismatch were assembled according to their overlap sequence. The sequences were then clustered into operational taxonomic units (OTUs) based on $97 \%$ identity using QIIME ${ }^{1}$. The representative sequences for each OTU were aligned to identify the species using PyNAST in QIIME. Rarefaction curves for alpha diversity were generated to assess the efficiency of the sequencing depth for representing and comparing microbial communities. Species richness was estimated using Chao-1 (Faith and Baker, 2006). The beta diversity of the microbial communities was determined by visual assessment using principle coordinate analysis (PCoA) plots and by an analysis of similarity calculated based on weighted UniFrac distances (QIIME) according to one-way non-parametric multivariate analysis of variance.

\footnotetext{
${ }^{1}$ http://qiime.sourceforge.net/
} 


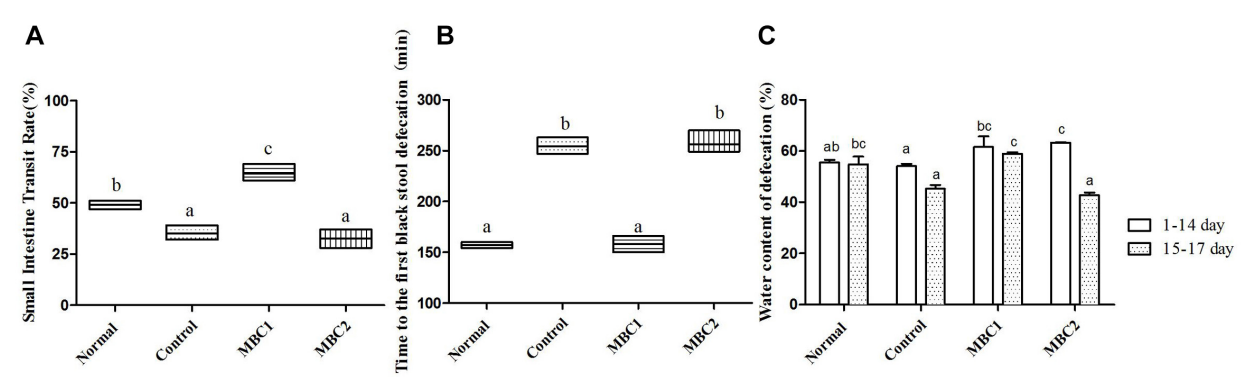

FIGURE 1 | Defecation status of mice during the experiments. (A) Small intestinal transit rate; (B) Time to the first black stool defecation; (C) water content of defecation; a-c, mean values with different letters over the bars are significantly different $(\rho<0.05)$ according to Duncan's multiple range test.

A

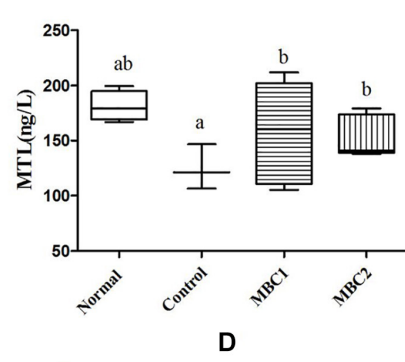

B

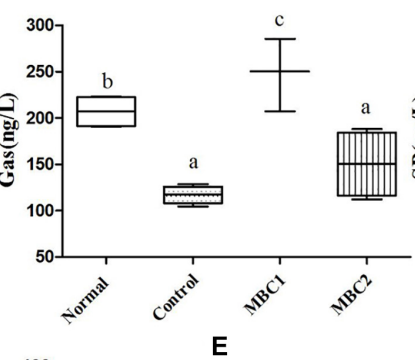

C
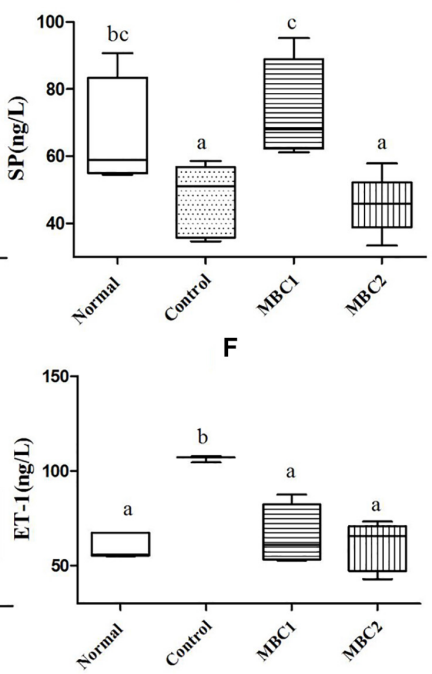

FIGURE 2 | Effects of CMB on constipation-related GI neurotransmitters. A, MTL; B, Gas; C, SP; D, SS; E, VIP; F, ET-1; a-C, mean values with different letters over the bars are significantly different $(p<0.05)$ according to Duncan's multiple range test.

\section{Statistical Analysis}

The data were presented as mean \pm SD and analyzed using GraphPad Prism 5 and Origin 8.5. The differences between the samples were analyzed by one-way ANOVA with Duncan's multiple range test. The results were considered significant when $p<0.05$.

\section{RESULTS}

\section{Bifidobacterium Combination With Adhesion Properties Significantly Improved the Symptoms of Constipation}

Before constipation was induced, the water content of the feces was higher in CMB1, and CMB2 groups than in the control and normal groups (Figure 1C, $p<0.05$ ). With the intake of loperamide, the fecal water content showed a downward trend in all groups, which indicated that loperamide induced constipation in mice.
Compared with the constipation group, the symptoms of constipation (i.e., the water content of the feces, the small intestinal transit rate and the first black stool defecation time) were significantly improved in CMB1 group (Figure 1, $p<0.05$ ). Meanwhile, there was no statistical difference between the CMB2 and control groups. These results demonstrate that CMB2 had

TABLE 2 | SCFAs in feces before constipation.

\begin{tabular}{lrrrr}
\hline SCFAs, $\boldsymbol{\mu}$ mol/g & \multicolumn{1}{c}{ Normal } & Control & \multicolumn{1}{c}{ CMB1 } & \multicolumn{1}{c}{ CMB2 } \\
\hline Acetic acid & $13.71 \pm 1.33^{\mathrm{a}}$ & $13.32 \pm 2.19^{\mathrm{a}}$ & $24.62 \pm 0.67^{\mathrm{b}}$ & $28.59 \pm 0.3^{\mathrm{c}}$ \\
Propionic acid & $2.25 \pm 0.14^{\mathrm{a}}$ & $1.98 \pm 0.3^{\mathrm{a}}$ & $2.38 \pm 0.25^{\mathrm{a}}$ & $2.24 \pm 0.19^{\mathrm{a}}$ \\
Butyric acid & $5 \pm 0.11^{\mathrm{c}}$ & $4.51 \pm 0.4^{\mathrm{bc}}$ & $3.62 \pm 0.16^{\mathrm{a}}$ & $4.42 \pm 0.36^{\mathrm{b}}$ \\
Total acid & $20.95 \pm 1.57^{\mathrm{a}}$ & $19.72 \pm 2.11^{\mathrm{a}}$ & $30.62 \pm 0.99^{\mathrm{b}}$ & $35.34 \pm 0.86^{\mathrm{c}}$
\end{tabular}

CMB1 group: (combining multiple strains of Bifidobacterium with adhesion property), $1 \times 10^{10} \mathrm{CFU}$; CMB 2 group: (combining multiple strains of Bifidobacterium with no adhesion property), $1 \times 10^{10} \mathrm{CFU}$. $^{\mathrm{a}-\mathrm{c}}$ Mean values with different letters over bars are significantly different $(p<0.05)$ according to Duncan's multiple range test. 
no effect on constipation induced by loperamide. There was no significant difference in weight gain, feed and water consumption between the mice throughout the experiment. This indicated that constipation had no effect on the weight and appetite of mice.

\section{Bifidobacterium Combination With Adhesion Properties Significantly Improved the GI Neurotransmitters Levels in Serum}

Constipation-related neurotransmitters, such as MTL, Gas, SP, ET-1, SS and VIP, play an important role in regulating gastrointestinal motility. In addition, the secretion of GI neurotransmitters is different in disease and normal state. Therefore, we studied these GI neurotransmitters in serum of mice after treated with CMB. The results showed that CMB1 obviously increased the levels of MTL, Gas and SP and decreased the levels of SS, VIP and ET-1. The variation tendency of GI neurotransmitters in CMB2 group were the same as that in CMB1 group, except for Gas and SP (Figure 2, $p<0.05$ ). In CMB1treated group, the Bifidobacteria combination showed preferable effect in relieving constipation. These results indicated that the difference in relieving constipation between CMB1 and CMB2 may be related to the levels of Gas and SP in serum.

\section{Bifidobacterium Combination With Adhesion Properties Significantly Raised the Concentration of SCFAs in Fecal Samples}

The contents of each SCFA in the feces are shown in Tables 2, 3. Before constipation, acetate, propionate and butyrate levels were fairly steady in normal and constipation control groups, while the concentrations of acetate and total acids increased, and the level of butyrate decreased in CMB1 and CMB2 groups. After the induction of constipation, the concentrations of acetate showed the same level in normal and control groups. Only propionic, butyric and total acids decreased compared to normal group. In addition, acetic, propionic and butyric acid concentrations increased in CMB1 group, whereas there was no significant difference between control and CMB2 groups in the concentrations of propionic and butyric acids. These results indicated that increasing the concentration of propionic and

TABLE 3 | SCFAs in feces after constipation.

\begin{tabular}{|c|c|c|c|c|}
\hline CFAs, $\mu \mathrm{mol} / \mathrm{g}$ & Normal & Control & CMB1 & \\
\hline & $13.85 \pm 1.15^{a}$ & $12.57 \pm 0$ & $35.9 \pm 2.29^{c}$ & $20.45 \pm$ \\
\hline opionic acid & $2.20 \pm 0.19^{b}$ & $1.68 \pm 0.3^{a}$ & $3.37 \pm 0.28^{c}$ & 1.88 \\
\hline & $4.69 \pm 0.66^{b}$ & $1.67 \pm 0.32^{a}$ & $4.46 \pm$ & \\
\hline & $20.74 \pm 1.71^{b}$ & $15.92 \pm 0.80^{a}$ & $2.76^{c}$ & \\
\hline \multicolumn{5}{|c|}{$\begin{array}{l}\text { CMB1 group: (combining multiple strains of Bifidobacterium with adhesion } \\
\text { property), } 1 \times 10^{10} \mathrm{CFU} \text {; CMB2 group: (combining multiple strains of } \\
\text { Bifidobacterium with no adhesion property), } 1 \times 10^{10} \mathrm{CFU} \text {; }{ }^{a-c} \text { Mean values with } \\
\text { different letters over bars are significantly different }(p<0.05) \text { according to Duncan's } \\
\text { multiple range test. }\end{array}$} \\
\hline
\end{tabular}

butyric acids in feces might be associated with the relief of constipation symptoms.

\section{Adhesive Bifidobacterium Combination Affected Fecal Microbiota in Different Way to Non-adherent Bifidobacterium Combination}

To evaluate the influences of the CMB on the intestinal flora, we analyzed the fecal samples of healthy and constipated BALB/C mice gavaged with $\mathrm{CMB} 1$ and $\mathrm{CMB} 2$ for 17 days. A dataset containing 325,440 high-quality sortable $16 \mathrm{~S}$ rDNA gene orders was produced from 32 fecal specimens using MiSeq sequencing. The average sequence read was 10,170 per sample. Representative sequences of all of the sequences were clustered, and a 97\% sequence similarity cut-off was used. The number of OTUs per sample ranged between 433 and 1952. We performed association tests based on $\alpha$ - and $\beta$ - diversity measures. There was a remarkable difference in $\alpha$-diversity between the normal and constipation control groups (Supplementary Figure S2). After induced constipation by loperamide, the $\alpha$-diversity indices (Chao-1, observed species and phylogenetic diversity) shown a sharp declined in constipation control and CMB2 groups. In contrast, CMB1 obviously increased the $\alpha$-diversity indices. It indicated that CMB1 significantly increased the taxa richness of fecal microbiota, while CMB2 had no effect on the decrease of fecal microbial diversity caused by constipation.

The $\beta$-diversity of the fecal flora in mice given CMB1 and CMB2 was revealed by using unweighted UniFrac matrixes. As shown in Supplementary Figure S3, the light blue symbol represents the normal group and was located at the lower section of the PCA diagram, while the red symbol represents the constipation control group and was shift from the lower section of the score plot to the left, indicating that constipation changed the fecal microbiota structure of mice. The green and dark blue symbols, respectively, represent the CMB1 and CMB2 groups and were, respectively, located at the upper middle and upper right section of the PCA diagram, indicating that CMB significantly changed the fecal microbiota structure of mice. However, CMB1 and CMB2 affected the fecal microbiota through absolutely different ways.

A total of 30,627 sequences were appointed to 1,952 OTUs, which agglomerated into 41 genera and 8 phyla using the Ribosomal Database Project (RDP) classifier. Changes in the taxa that were obviously different between treatments are shown in Figures 3-5.

\section{Actinobacteria}

Two genera (Bifidobacterium and Adlercreutzia) were included in this phylum. At the genus level, the level of Bifidobacterium in $\mathrm{CMB}$ groups were significantly higher than in constipation group. There was no significant difference among groups in the level of Adlercreutzia (Figrue 3, Bifidobacterium).

\section{Bacteroidetes}

Seven genera Bacteroides, Odoribacter, Parabacteroides, Barnesiella, Rikenella, Prevotella and Alistipes were included 


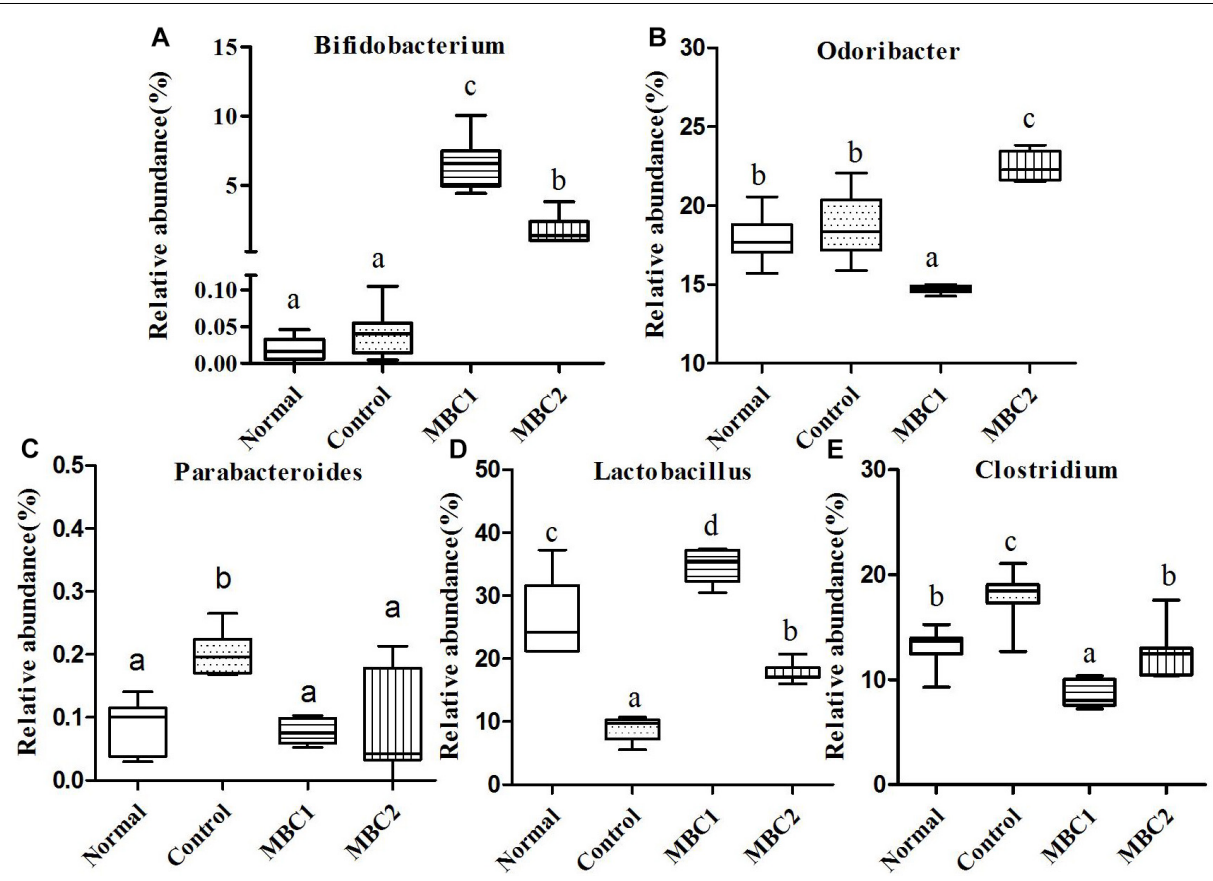

FIGURE 3 | Relative abundance (\%) of fecal microbial taxa at the genus level in constipated mice fed CMB. (A) Bifidobacterium, (B) Odoribacter, (C) Parabacteroides, (D) Lactobacillus, and (E) Clostridium.

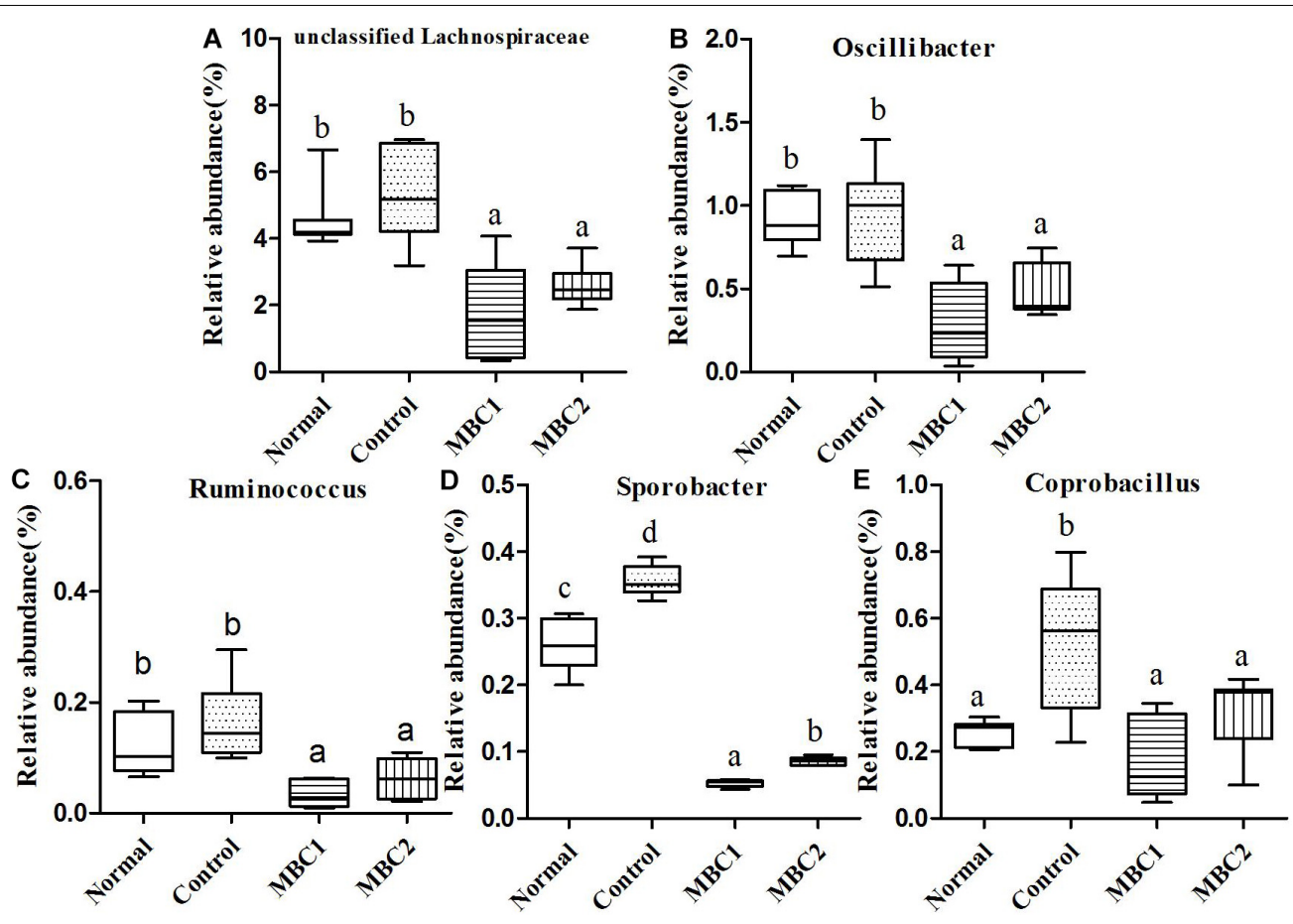

FIGURE 4 | Relative abundance (\%) of fecal microbial taxa at the genus level in constipated mice fed CMB. (A) Unclassified Lachnospiraceae, (B) Odoribacter, (C) Ruminococcus), (D) Sporobacter, and (E) Coprobacillus.

in this phylum. Odoribacter was the lowest in CMB1 group, followed by normal and control groups and then CMB2 group. The relative abundance of Parabacteroides decreased to normal level after treated with CMB. The relative abundance of Prevotella recovered to normal level in CMB1 treatment group, whereas there was no significant 


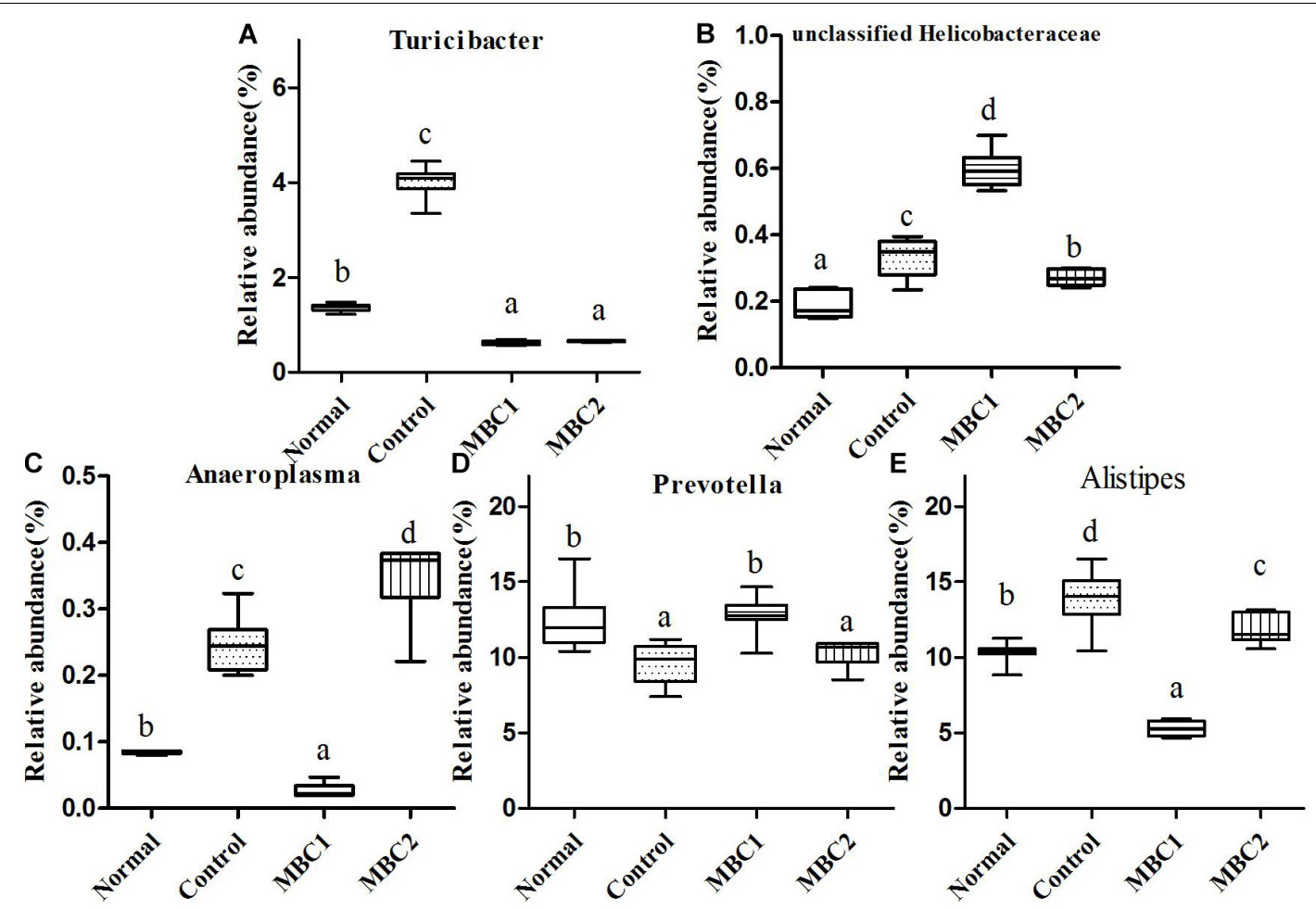

FIGURE 5 | Relative abundance (\%) of fecal microbial taxa at the genus level in constipated mice fed CMB. (A) Turicibacter, (B) unclassified Helicobacteraceae, (C) Anaeroplasma, (D) Prevotella and (E) Alistipes.

difference between CMB2 and constipation control group. Alistipes was the lowest in CMB1 treatment group and the highest in constipation control group. There was no significant difference among groups in the level of other genera (Figrues 3, 5, Odoribacter, Parabacteroides, Prevotella and Alistipes).

\section{Deferribacteres}

This phylum included a single genus, Mucispirillum. There was no significant difference among groups in the level of Mucispirillum.

\section{Firmicutes}

Twenty-seven genera (such as Staphylococcus, Lactobacillus, Lactococcus, Streptococcus, Clostridium, Eubacterium, Blautia, Dorea, Oscillibacter, Ruminococcus, Sporobacter, Coprobacillus, Turicibacter and so on) were included in this phylum. At the genus level, the relative abundance of unclassified Lachnospiraceae, Clostridium, Sporobacter, Coprobacillus, Turicibacter, Oscillibacter and Ruminococcus decreased significantly in $\mathrm{CMB}$ groups compared to control group. Meanwhile, the relative abundance of Clostridium and Sporobacter were higher in CMB2 treatment group than that in CMB1 group. In contrast, the relative abundance of Lactobacillus was the highest in CMB1 treatment group, followed by normal and CMB2 groups and then the constipation control group (Figures 3-5). There was no significant difference among groups in the level of other genera.

\section{Proteobacteria}

Only one genus was found in this phylum. The relative abundance of unclassified Helicobacteraceae only increased in CMB1 treatment group, whereas there was no significant difference among other groups (Figure 5).

\section{TM7}

This phylum contained one genus, the unclassified TM7. There was no significant difference among groups in this genus.

\section{Tenericutes}

Anaeroplasma was the only genus found in this phylum. Its level was highest in CMB2 group followed by the control and normal groups and then the CMB1 group (Figure 5, Anaeroplasma).

\section{Verrucomicrobia}

Akkermansia was the only genus found in this phylum. Its level was only increased in CMB1 treatment group, whereas there was no significant difference among other groups.

In brief, in CMB1-treated group, the relative abundances of Bifidobacterium, Lactobacillus, Akkermansia, Prevotella and unclassified Helicobacteraceae significantly increased and the levels of Clostridium and Anaeroplasma in feces samples decreased compared to CMB2-treated group. Moreover, the levels of Bifidobacterium, Lactobacillus, Akkermansia and Prevotella were negatively correlated with constipation, while Clostridium and Anaeroplasma were positively correlated with constipation. Therefore, after CMB1 

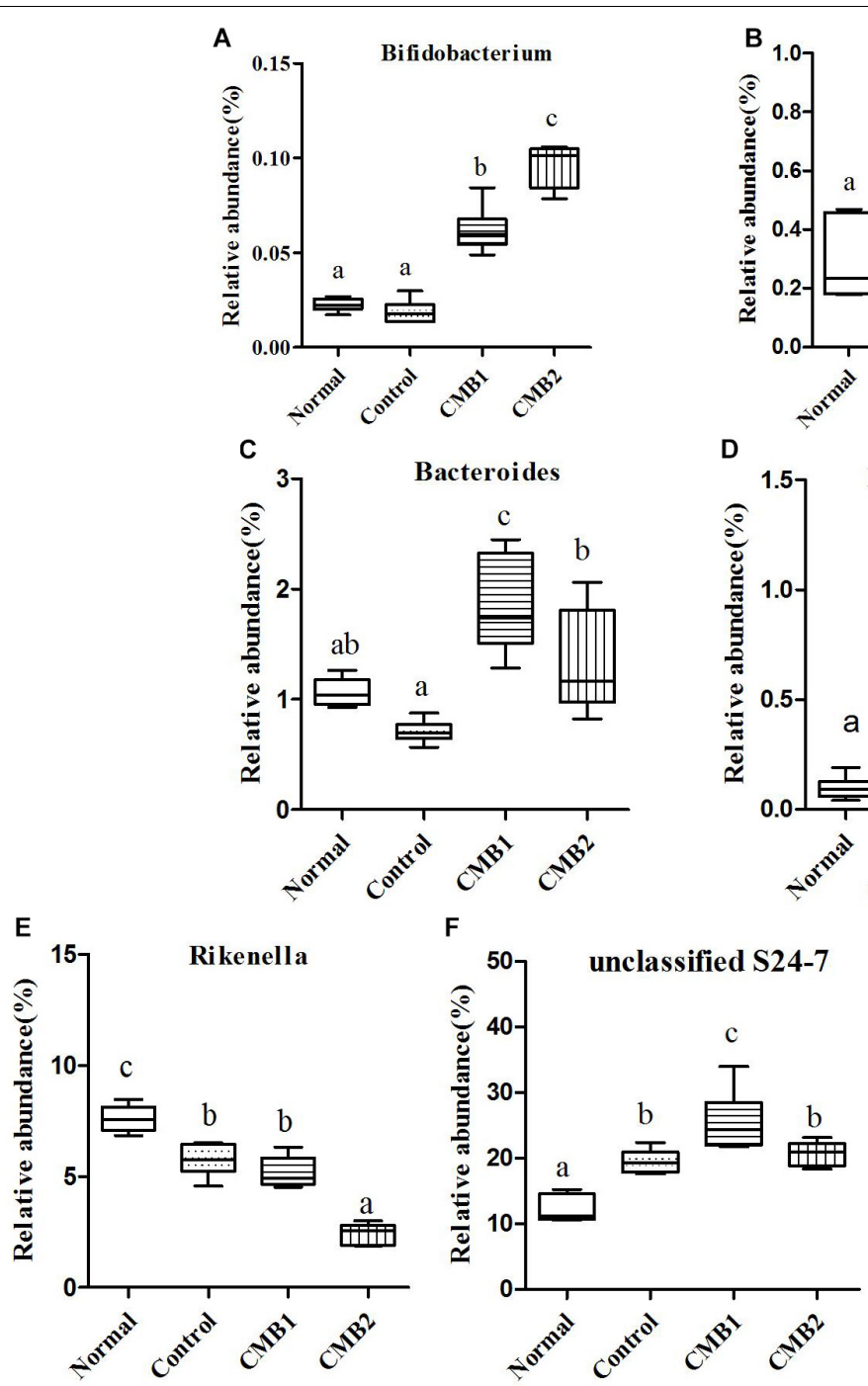
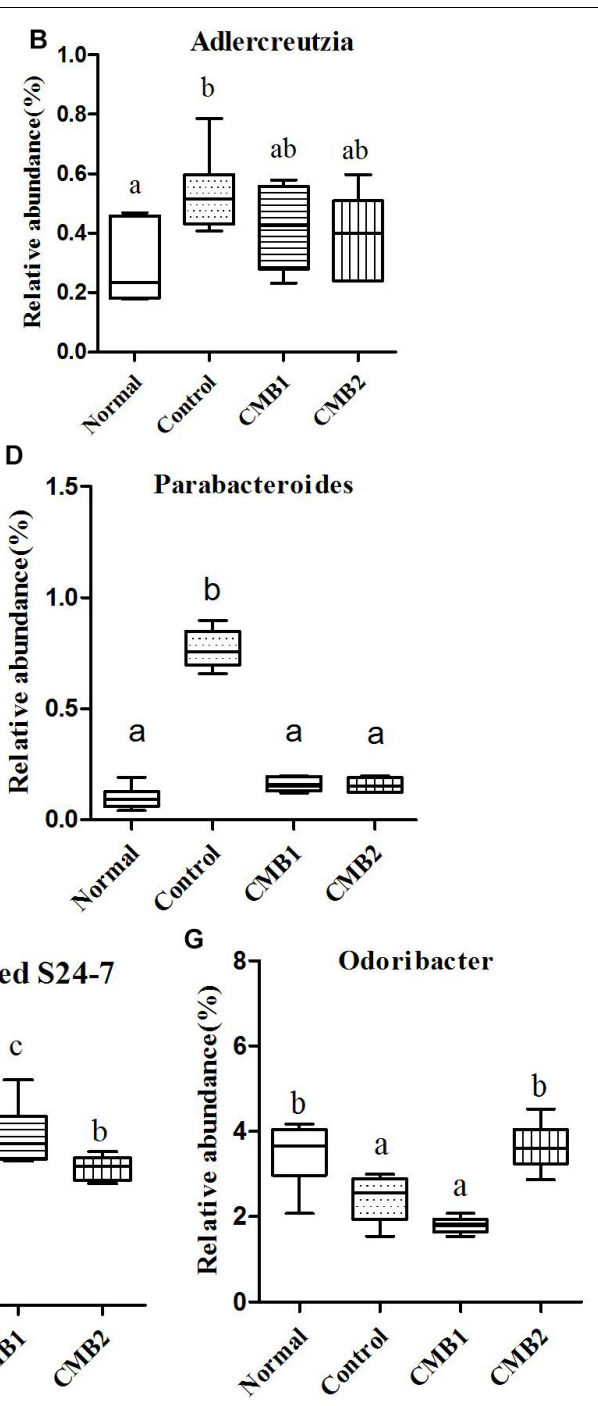

FIGURE 6 | Relative abundance (\%) of caecal microbial taxa at the genus level in constipated mice fed CMB. (A) Bifidobacterium, (B) Adlercreutzia, (C) Bacteroides, (D) Parabacteroides, (E) Rikenalla, (F) Unclassified S24-7, and (G) Odoribacter.

intervention, the gut microbiota in constipated mice tended to be beneficial to host health.

\section{Adhesive Bifidobacterium Combination Affected Caecal Microbiota in Different Way to Non-adherent Bifidobacterium Combination}

A dataset containing 373,216 high-quality sortable $16 \mathrm{~S}$ rDNA gene orders was produced from 32 fecal specimens using MiSeq sequencing. The average sequence read was 11,663 per sample. Representative sequences of all of the sequences were clustered, and a $97 \%$ sequence similarity cut-off was used. The number of OTUs per sample ranged between 412 and 1330. We performed association tests based on $\alpha$ - and $\beta$ - diversity measures. The normal and constipation control groups had obvious differences in $\alpha$ - diversity based on species richness, the observed species and diversity (Supplementary Figure S4). After CMB treatment, the chao-1 index and observed species showed significant changes in CMB1 group. The phylogenetic diversity index was obviously changed in CMB1 and CMB2 groups compared with the constipation group. The $\beta$ diversity of the caecal microbiota was assessed using unweighted UniFrac matrixes. As shown in Supplementary Figure S5, constipation changed the caecal microbiota structure of mice greatly, while CMB significantly changed the caecal microbiota structure of mice. Different from the absolutely different ways by $\mathrm{CMB} 1$ and $\mathrm{CMB} 2$, respectively, these two Bifidobacterium combinations showed a certain overlap in the caecal microbiota regulation.

A total of 29,193 sequences were assigned to 1,396 OTUs that were clustered into 54 genera and 9 phyla using the RDP classifier. Changes in taxa are shown in Figures 6-8. 


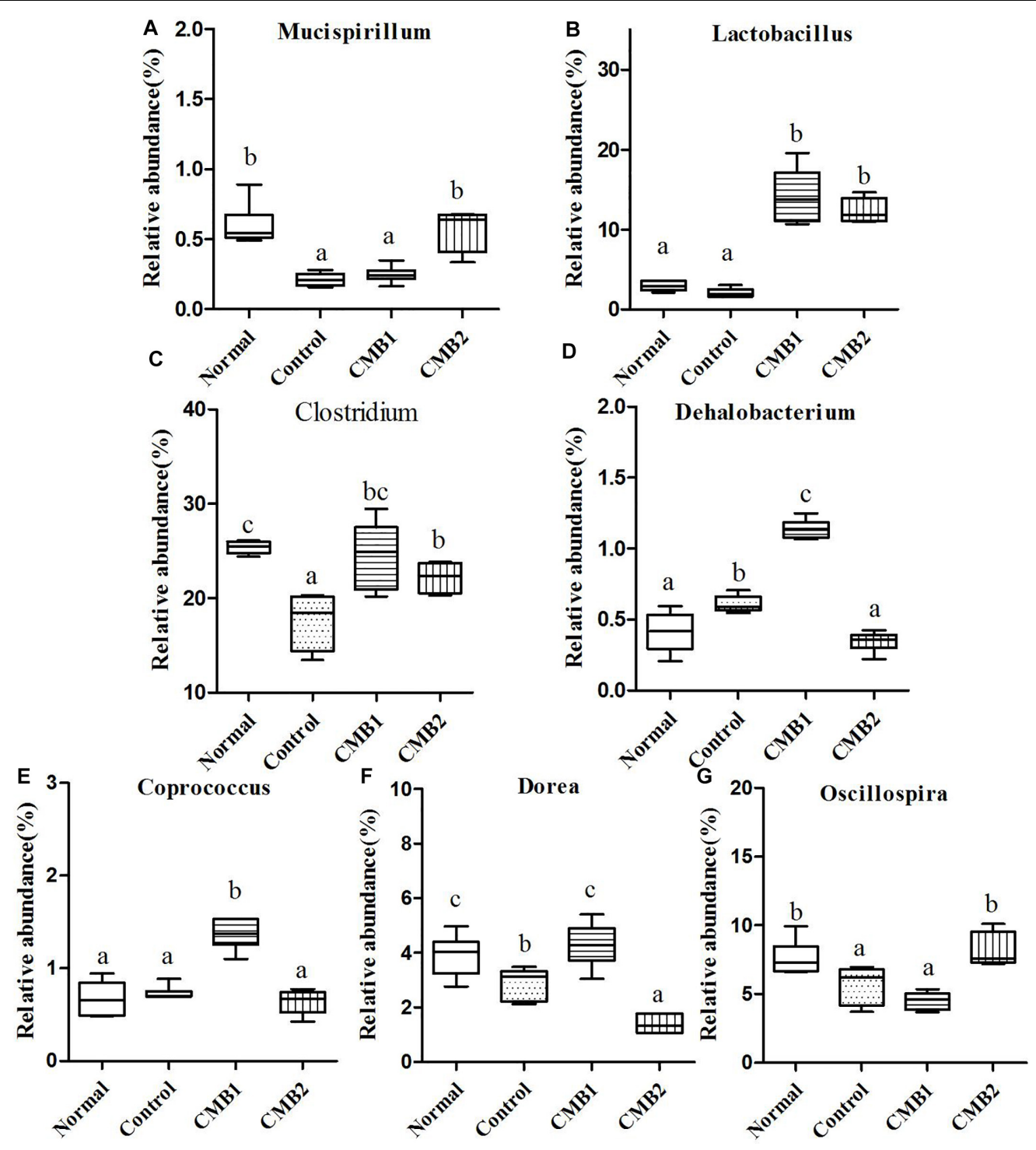

FIGURE 7 | Relative abundance (\%) of caecal microbial taxa at the genus level in constipated mice fed CMB. (A) Mucispirillum, (B) Lactobacillus, (C) Clostridium, (D) Dehalobacterium, (E) Coprococcus, (F) Dorea, and (G) Oscillospira.

\section{Actinobacteria}

Two genera were found in this phylum, Adlercreutzia only increased in control group, whereas there was no significant difference among other groups. The relative abundance of Bifidobacterium was the highest in CMB2 treatment group, followed by CMB1 treatment group and then normal and control groups (Figure 6, Bifidobacterium).

\section{Bacteroidetes}

Twelve genera were found in this phylum. At the genus level, the level of Bacteroides was the highest in CMB1 treatment group, followed by $\mathrm{CMB} 2$ and then the normal and control groups. The levels of Parabacteroides only increased significantly in control group, whereas there was no significant difference among other groups. The level of Rikenella was the highest in CMB1 group and the lowest in CMB2 treatment group, whereas there was no significant difference between normal and control group. The relative abundance of unclassified S24-7 was the highest in CMB1 treatment group, followed by CMB2 and control groups and then the normal group. The relative abundance of Odoribacter recovered to normal level after treated with $\mathrm{CMB} 2$, whereas there was no significant difference between CMB1 and control group. There was no significant difference among groups in the level of other genera (Figure 6).

\section{Cyanobacteria}

Unclassified YS2 was the only genus found in this phylum, and there was no significant difference among groups in this genus. 


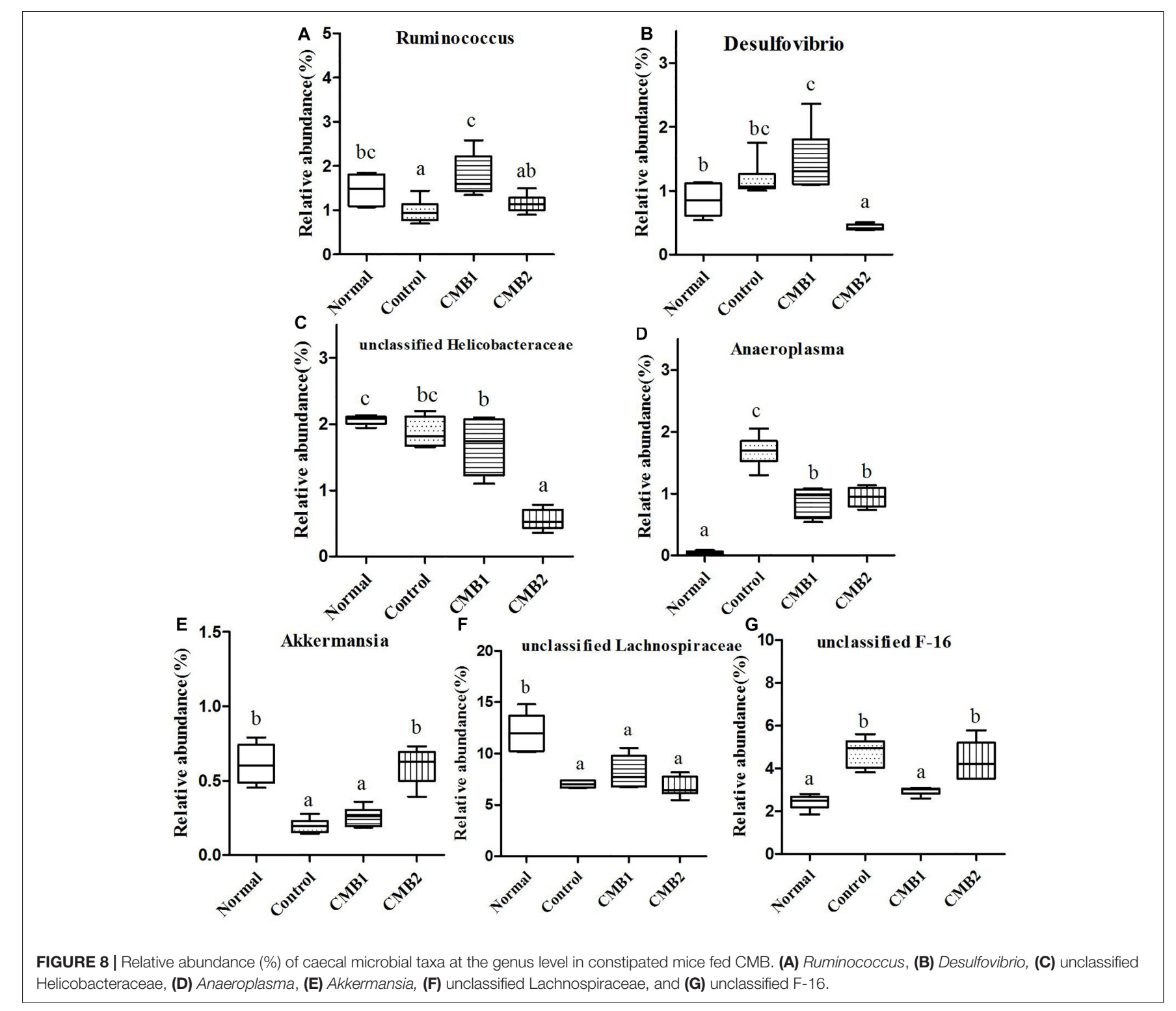

\section{Deferribacteres}

Mucispirillum was the only genus found in this phylum, and the relative abundance of Mucispirillum recovered to normal level after treated with CMB2, whereas there was no significant difference between CMB1 and control group (Figure 7).

\section{Firmicutes}

Twenty-six genera (such as Lactobacillus, unclassified Clostridiales, Clostridium, Dehalobacterium, unclassified Lachnospiraceae, Coprococcus, Dorea, unclassified Ruminococcaceae, Oscillospira, Ruminococcus and so on) were found in this phylum. The abundances of Dehalobacterium, Dorea, Coprococcus, and Ruminococcus were significantly higher in CMB1group compared to CMB2 group, whereas the abundance of Oscillospira was obviously lower in CMBlgroup compared to CMB2 group. The relative abundance of Lactobacillus, and Clostridium increased significantly in
CMB groups compared to control group. The relative abundance of unclassified Lachnospiraceae decreased significantly in all treatment groups, except the normal group. And there was no significant difference among groups in the level of other genera (Figures 7, 8).

\section{Proteobacteria}

Seven genera were found in this phylum. The level of Desulfovibrio was the highest in CMB1 group and the lowest in CMB2 treatment group, whereas there was no significant difference between other groups. Meanwhile, the level of unclassified Helicobacteraceae was the lowest in CMB2 treatment group, and the highest in normal group, whereas there was no significant difference between control and CMB2 treatment group. There was no significant difference among groups in the level of other genera (Figures 7, 8). 


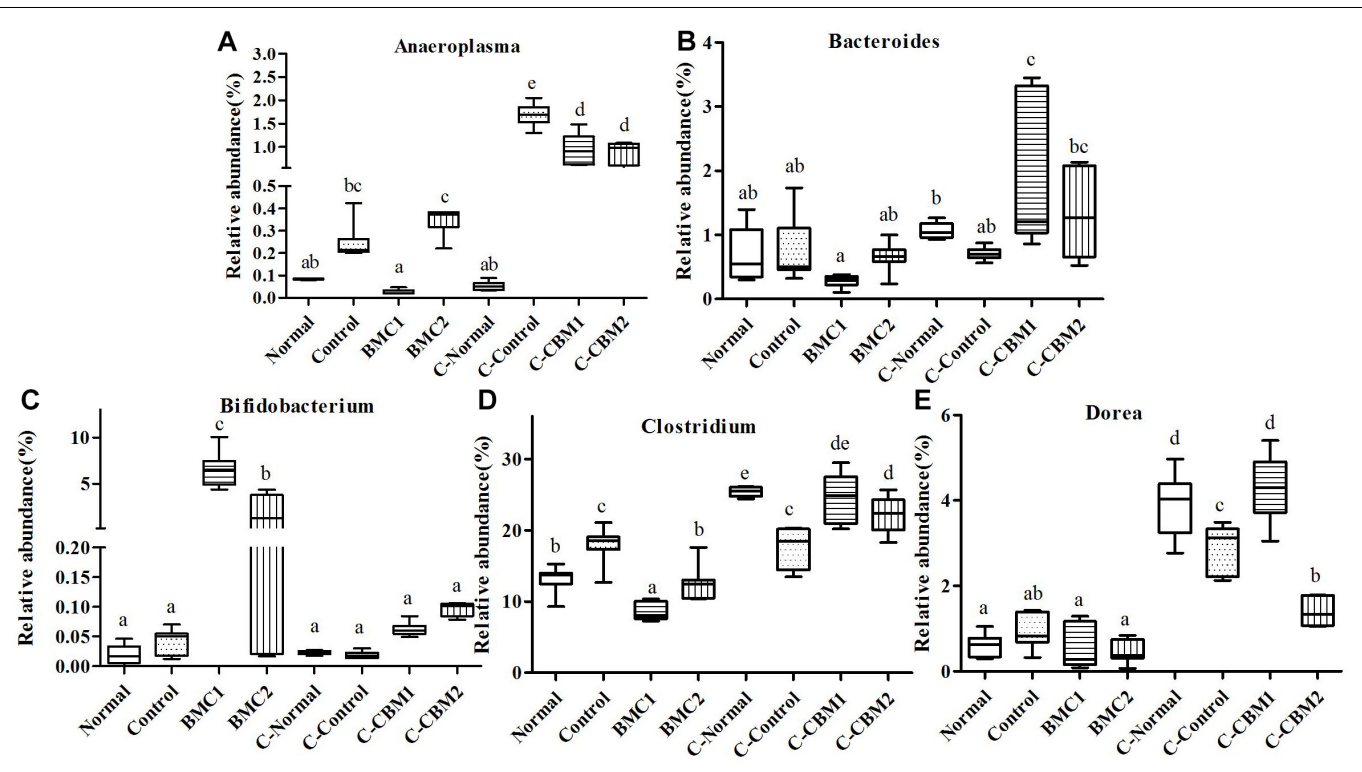

FIGURE 9 | The genus-level comparison of fecal samples and caecum content samples. (A) Anaeroplasma, (B) Bacteroides, (C) Bifidobacterium, (D) Clostridium, and (E) Dorea.

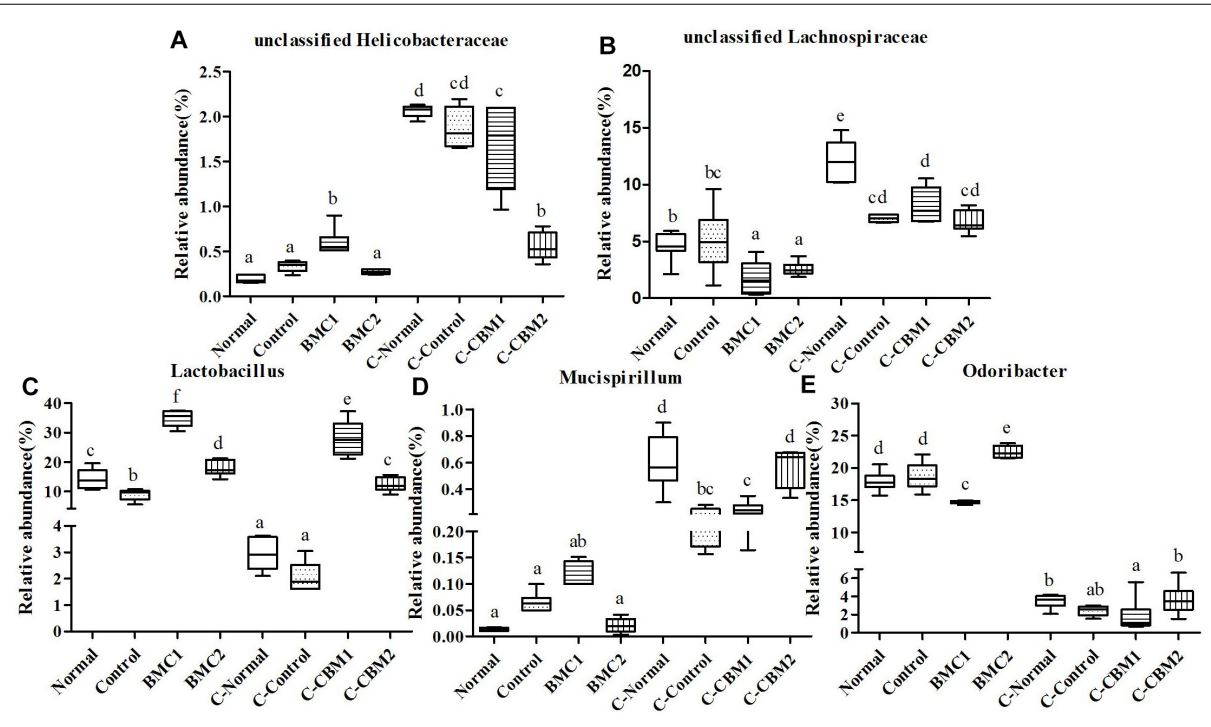

FIGURE 10 | The genus-level comparison of fecal samples and caecum content samples. (A) Unclassified Helicobacteraceae, (B) unclassified Lachnospiraceae, (C) Lactobacillus, (D) Mucispirillum, and (E) Odoribacter.

TM7

This phylum included one genus, unclassified F16. Its level was decreased to normal group in CMB1 treatment group, whereas there was no significant difference between CMB2 and control group (Figure 8).

\section{Tenericutes}

This phylum included one genus. The relative abundance of Anaeroplasma was the highest in control group, followed by $\mathrm{CMB}$ treatment groups and then the normal group (Figure 8).

\section{Verrucomicrobia}

Akkermansia was the only genus found in this phylum. Its level was only increased in CMB treatment group, whereas there was no significant difference between normal and control group (Figure 8).

In brief, after CMB intervention, the common effect of CMB1 and $\mathrm{CMB} 2$ on caecum microbiota was that they significantly elevated the relative abundances of Lactobacillus, Clostridium, Akkermansia and Bifidobacterium and reduced the relative abundances of Anaeroplasma. The difference was that in CMB1-treated group, the relative abundances of Bacteroides, 


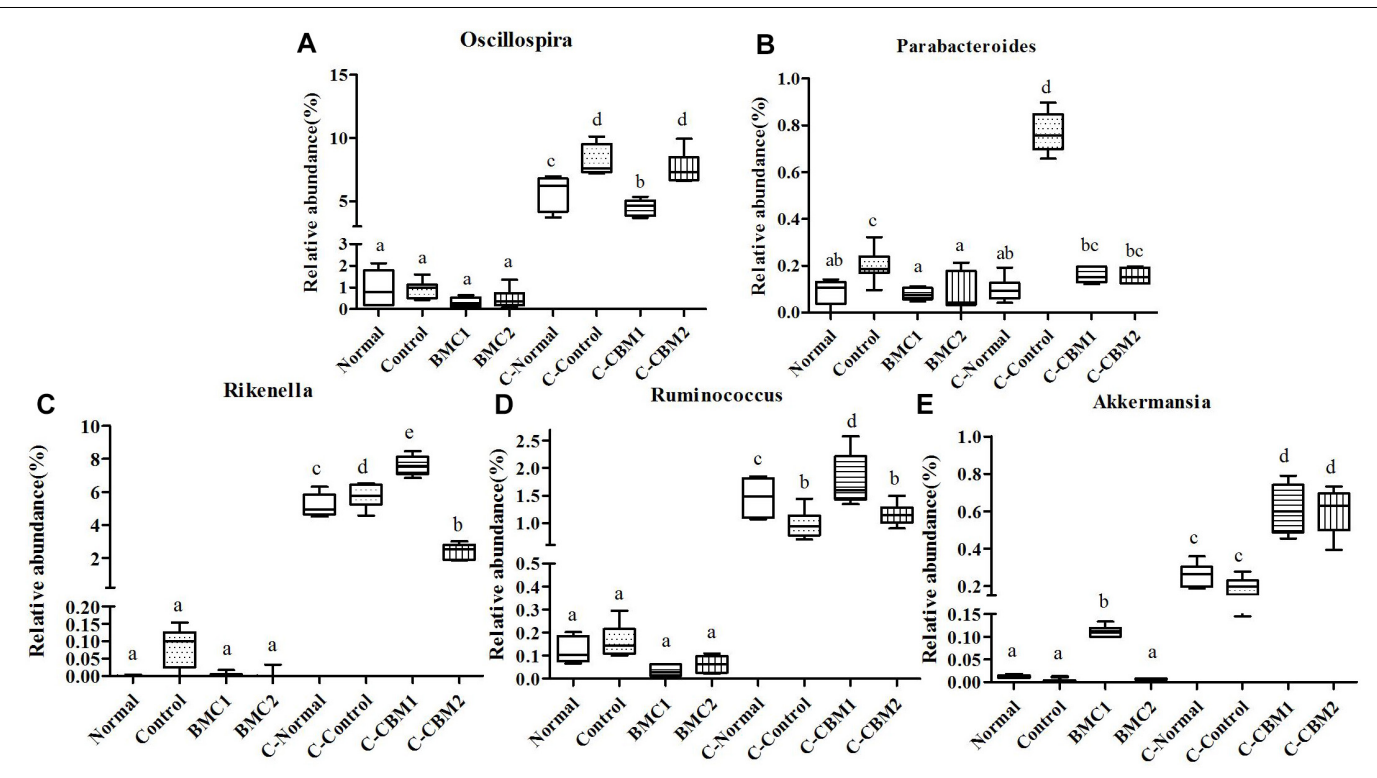

FIGURE 11 | The genus-level comparison of fecal samples and caecum content samples. (A) Oscillospira, (B) Parabacteroides, (C) Rikenella, (D) Ruminococcus, and (E) Akkermansia.

Rikenella, unclassified S24-7, Dorea, Coprococcus, Ruminococcus, Dehalobacterium and Desulfovibrio significantly increased and the relative abundances of Oscillospira, Odoribacter, Mucispirillum and unclassified F16 decreased, while all these changes were opposite in CMB2-treated group. Considering the result that $\mathrm{CMB1}$ alleviated constipation effectively, it seemed that the differences on cecum microbiota between CMB1- and CMB2- treated groups might be closely related to the situation of constipation in mice.

Comparing the fecal flora with the cecum content flora at the genus level, we found that there were 15 genera with significant differences between these two samples. As shown in Supplementary Figure S6. the fecal microbiota was located at the right of the score plot, whereas the caecal microbiota was located at the left of the score plot. It indicated that there were significant differences between fecal microbiota and caecum microbiota. Differences in taxa are shown in Figures 9-11.

At the genus level, there were 15 genera with significant differences between fecal microbiota and caecum microbiota, and 11 of these 15 genera showed that the relative abundance of caecum samples was significantly higher than that of fecal samples. In contrary, the relative abundances of Lactobacillus, Bifidobacterium, and Odoribacter were higher in fecal samples than in cecum samples (Figures 9, 10). In addition, the relative abundances of Bacteroides and Clostridium in feces sample and caecum sample presented the opposite tendency in CMB1 group, whereas the level of Anaeroplasma showed the same tendency (Figure 9). The relative abundances of Dorea, Rikenella and Ruminococcus in cecum sample increased obviously in CMB1treated group, whereas the level of Oscillospira in cecum sample decreased obviously in CMB1-treated group. And the level of Odoribacter in fecal sample decreased obviously in CMB1-treated group (Figures 9-11).

\section{DISCUSSION}

Although many efforts have been made to treat constipation, it remains one of the most common diseases worldwide. Therefore, it is urgent to explore an effective, safe and low-toxicity methods to treat constipation. Clinical studies have found that gut microbiota plays an important role in the occurrence and development of constipation. Thus, micro-ecological therapy has gradually replaced the traditional treatment for constipation.

A large number of animal and human experiments have confirmed that probiotics can alleviate constipation (Bu et al., 2007; Tabbers et al., 2011; Urita et al., 2015). However, the adhesion property of strains is the premise of this study in evaluating the probiotic effect of strains. Therefore, in this study, ten strains of Bifidobacteria were divided into two groups according to their adhesion property to investigate their efficacy in alleviating constipation. The results showed that CMB1 (combination of the adhesive Bifidobacteria) significantly improved the water content of feces, the small intestinal transit rate and the first black stool defecation time, whereas the CMB2 (combination of non-adhesive Bifidobacteria) had no effect on constipation. This result was consistent with our previous study (Wang et al., 2017a). The results showed that whether a single strain or multiple strains of Bifidobacterium are used, the effect of relieving constipation is related to the adhesion property of the bacteria.

The known factors affecting intestinal motility are nervous systems (intestinal nervous system and autonomic nervous system), immune system, intestinal microbiota and their metabolites. Any disorder or dysfunction in any of these factors can lead to intestinal motility disorders (constipation or diarrhea). Therefore, in this study, GI neurotransmitters levels were measured to determine the 
effect of Bifidobacteria on constipation-related intestinal nervous activities. The results showed that the reduced GI neurotransmitters in serum of constipation mice tended to be up-regulated in CMB1 intervention group, while CMB2 intervention had no effect on recovery of neurotransmitters (MTL, Gas and SP). Thus, CMB1 may alleviate constipation by up-regulating neurotransmitters (MTL, Gas and SP). Considering the adhesion properties of Bifidobacterium, we believe that the bacteria with adhesion property may have a greater chance of establishing close contact with and colonizing the intestine to exert GI neurotransmitter regulatory effects.

It has been suggested that the changes of gut flora and metabolites may be the important reasons for the pathophysiological changes in constipation. Several previous studies have reported that an increased concentration of SCFAs in the intestine is beneficial to constipation (Salminen and Salminen, 1997; Veiga et al., 2014). SCFAs are produced by the bacterial fermentation of dietary fiber. They are reported to have important physiological effects in the intestine. For example, SCFAs influence the functions of the gastrointestinal tract (Ríos-Covián et al., 2016), electrolyte balance (Vidyasagar and Ramakrishna, 2000), and ion transport (Yajima, 1988). The concentration of SCFAs in feces reflects the activity of enteric microorganisms and is important in the relief of constipation by CMB. Our study showed that CMB1 improved the symptoms of constipation and increased the levels of propionic and butyric acids. This increase corresponded to an increase in propionic and butyric acid producing bacteria. Propionic acid producing bacteria are mainly Bacteroidetes, and butyric acid levels are positively correlated with the relative abundance of Firmicutes (Guilloteau et al., 2010). In our study the observed increases in propionic and butyric acid are consistent with an increase in Prevotella and Lactobacillus in feces samples treated with CMB1, and increase in unclassified S247, Rikenella, Bacteroides, Dorea, Lactobacillus, Dehalobacterium, Desulfovibrio, Ruminococcus and Coprococcus in caecal content samples treated with CMB1. It has been widely reported that either Prevotella or Bacteroides dominates the human gut and they were proposed to be antagonistic (Choi and Chang, 2015). Prevotella and Bacteroides, which are thought to have had a common ancestor, benefited their host by excluding potential pathogens from colonizing the gut (Ley, 2016). It also can be seen from the results that the ratio of Firmicutes to Bacteroidetes increased significantly in feces samples of CMB1-treated group (from 0.89 in control group to 1.55 in CMB1group). A higher ratio of Firmicutes to Bacteroidetes is associated with faster transit in the large intestine (Sadik et al., 2004; DelgadoAros et al., 2008). Meanwhile, as observed in this study, the higher abundance of Actinobacteria (Bifidobacterium) in feces sample of CMB1-treated group was also correlated with faster colonic transit and in agreement with an earlier study (Parthasarathy et al., 2016). Therefore, it seems that the fecal microbiota profile may be related to colonic transit.

After treatment with CMB1, the genera unclassified S24-7, Rikenella, Bacteroides, Dorea, Lactobacillus, Dehalobacterium,
Desulfovibrio, Ruminococcus and Coprococcus increased in caecal content sample. Unclassified $\mathrm{S} 24-7$ is a prevalent and abundant bacterial component of the gut microbiome of mammals (Ormerod et al., 2016) and Unclassified S24-7 is more abundant following treatment-induced remission of colitis in mice (Rooks et al., 2014). The genera Firmicutes-Dorea and Bacteroidetes-Bacteroides were correlated inversely with the production of methane, while the Firmicutes-Oscillospira and Bacteroidetesodoribacter were correlated directly with the production of methane (Parthasarathy et al., 2016). In our research, the relative abundances of Dorea and Bacteroides increased significantly and the genera of Odoribacter and Oscillospira decreased obviously in caecal microbiota, thus suggest that the caecal microbiota is a better indicator for constipation and methane production than fecal microbiota. Furthermore, the genera Firmicutes-Coprococcus and Firmicutes-Lactobacillus which were correlated directly with colonic transit (Parthasarathy et al., 2016), increased significantly in caecum microbiota. These findings suggest that the caecal microbiota is also a better indicator for colonic transit. Taken together, compared with fecal microbiota, cecum microbiota reflects constipation state more comprehensively and accurately.

As mentioned above, adhesive Bifidobacterium combination affected fecal and caecal microbiota with different way to nonadherent Bifidobacterium combination. Furthermore, changes in the caecal contents microbiota and fecal microbiota were different as well whether treated with adhesive or nonadherent Bifidobacterium. It was found that there were seven genera with significant differences between fecal microbiota and caecal content microbiota. Meanwhile, six of these seven genera showed a high abundance in the caecal content compared to the relative abundance in the feces, whether in the CMB1 treatment group or the CMB2 treatment group. Such as the relative abundances of Oscillibacter, Clostridium, Anaeroplasma, Ruminococcus and so on. This might be due to the different environments in different part of intestine which resulted in different abundance of intestinal microbes. The intake of Bifidobacterium affected the microbiota in these parts of intestine. Due to the differences of microbiota species abundance, the influences by Bifidobacterium on caecal contents microbiota and fecal microbiota were different as well. We believe that adhesive Bifidobacteria can not only stay in the intestinal tract for a long time, but also produce more metabolites, so as to play a more effective role in changing gut microbiota than that by nonadhesive Bifidobacteria.

In this study, the relative abundances of Bifidobacterium (Actinobacteria), Lactobacillus (Firmicutes) and Akkermansia (Verrucomicrobia), which have been shown to improve the motility of the intestine by provoking the release of 5-HT or by promoting cholinergic pathways (Reigstad et al., 2015), were associated with faster transit in the large intestine. It is also consistent with our previous study (Wang et al., 2017a) where feeding constipated mice with CMB regulated the dysbiosis of the gut microbiota (decreases in the levels 
of Bifidobacterium and Lactobacillus) caused by constipation. Recent studies in rodents indicate that Akkermansia in the gut might reduce obesity, diabetes and inflammation (Everard et al., 2013). In this study, overgrowth of Bifidobacterium, Lactobacillus and Akkermansia was found to accompany reduction in the number of other bacteria, such as Anaeroplasma, Parabacteroides, Clostridium (in fecal samples) and unclassified Helicobacteraceae (only showed slight downward trend in the caecal contents sample). Some species of Anaeroplasma, Parabacteroides and Clostridium are pathogenic to humans and are associated with colitis and gastroenteritis (Ethan et al., 2011; Michael et al., 2016). Some species of Helicobacter (unclassified Helicobacteraceae) are associated with peptic ulcers, chronic gastritis, duodenitis and stomach cancer (Alexander et al., 2014; Watari et al., 2014; Seyed et al., 2015; Sostres et al., 2015). The genera Clostridium, Dorea, Oscillibacte, Ruminococcus, Sporobacter and Turicibacter are considered opportunistic pathogens (Sokol et al., 2008; Jenq et al., 2012; Konikoff and Gophna, 2016). When probiotics are predominated in the gut, opportunistic pathogens can have adjuvant effects, but when pathogenic bacteria are predominated, opportunistic pathogens can have pathogenic effects. In our present study, the overgrowth of probiotics changed the microenvironment of the gut and helped the opportunistic pathogens to develop in the health promoting direction. Thus, the changes in the above genera had a potential regulatory role in relieving constipation. It is certain that the intestinal microbiota showed dysbiosis after constipation. However, whether dysbiosis causes constipation or represents an epiphenomenon remains unclear. Further studies are needed to determine the true cause-andeffect relationship.

\section{CONCLUSION}

In conclusion, this study demonstrates that strains with adhesion properties (CMB1) can alleviate constipation more efficiently. CMB1 noticeably increased the water content of feces, small intestinal transit rates, the first black stool defecation time and the concentration of propionic and butyric acids. Moreover, constipated mice treated with CMB1 had a unique profile of caecal microbiota that reflect the relief of constipation more comprehensively and accurately, in comparison with fecal microbiota which was only associated with the colonic transit.

\section{REFERENCES}

Alexander, C. F., David, F., Forman, D., Hunt, R. H., Yuan, Y., and Moayyedi, P. (2014). Helicobacter pylori eradication therapy to prevent gastric cancer in healthy asymptomatic infected individuals: systematic review and meta-analysis of randomized controlled trials. BMJ. 348, 3174-3186. doi: 10.1136/bmj. g3174

Bharucha, A. E., Pemberton, J. H., and Locke, G. R. (2013). American gastroenterological association technical review on constipation. Gastroenterol. 144, 218-238. doi: 10.1053/j.gastro.2012.10.028

\section{DATA AVAILABILITY}

The datasets (gut metagenome Genome sequencing and assembly. SRA accession: PRJNA531550) for this study can be found in the [Sequence Read Archive (SRA)] (https://www.ncbi.nlm.nih.gov/bioproject/PRJNA531550).

\section{ETHICS STATEMENT}

This study was approved by the Ethics Committee of the Jiangnan University, China (JN. No. 20150326-0110-21) and performed at the Experimental Animal Center of the Jiangnan University [License No. SYXK(SU)2016-0045].

\section{AUTHOR CONTRIBUTIONS}

LW and GW conceived and designed the experiments. LW, CC, and SC performed the experiments. LW, GW, Y-kL, and $\mathrm{HZ}$ analyzed the data. JZ, HZ, and WC contributed reagents, materials, and analysis tools. LW, GW, and Y-kL wrote the manuscript. All authors contributed to the manuscript revision, and read and approved the submitted version.

\section{FUNDING}

This work was supported by the National Natural Science Foundation of China (Nos. 81800469, 31671839, and 31820103010), the Natural Science Foundation of Jiangsu Province (BK20180613), the Project funded by China Postdoctoral Science Foundation (2018M642164), the Postdoctoral Science Foundation of Jiangsu Province (2018K090C), the National Natural Science Foundation of China Key Program (No. 31530056), the National First-Class Discipline Program of Food Science and Technology (JUFSTR20180102), and the Program of Collaborative Innovation Center of Food Safety and Quality Control in Jiangsu Province.

\section{SUPPLEMENTARY MATERIAL}

The Supplementary Material for this article can be found online at: https://www.frontiersin.org/articles/10.3389/fmicb. 2019.01721/full\#supplementary-material

Bu, L. N., Chang, M. H., Yen-Hsuan, N. I., Chen, H. L., and Cheng, C. C. (2007). Lactobacillus casei rhamnosus Lcr35 in children with chronic constipation. Pediatr. Int. 49, 485-490. doi: 10.1111/j.1442-200X.2007.02397-x

Caesar, R., Tremaroli, V., Kovatcheva-Datchary, P., Cani, P., and Backhed, F. (2015). (2015). Cell Metabol. 22, 658-668. doi: 10.1016/j.cmet.2015. 07.026

Caporaso, J. G., Kuczynski, J., Stombaugh, J., Bittinger, K., Bushman, F. D., and Costello, E. K. (2010). QIIME allows analysis of high-throughput community sequencing data. Nat. Methods. 7, 335-336. doi: 10.1038/nmeth. f.303 
Chen, J., Euijung, R., Hathcock, M., Ballman, K., Chia, N., Olson, J. E., et al. (2016). Impact of demographics on human gut microbial diversity in a US Midwest population. Peer J. 4:e1514. doi: 10.7717/peerj.1514

Choi, C. H., and Chang, S. K. (2015). Alteration of gut microbiota and efficacy of probiotics in functional constipation. J. Neurogastroenterol. Motil. 21, 4-7. doi: $10.5056 /$ jnm 14142

DelgadoAros, S., Camilleri, M., Garcia, M. A., Burton, D., and Busciglio, I. (2008). High body mass alters colonic sensory-motor function and transit in humans. Am. J. Physiol. Gastrointest Liver Physiol. 295, G382-G388. doi: 10.1152/ajpgi. 90286.2008

Drouault, H. S., Bieuvelet, S., Burckel, A., Cazaubiel, M., Dray, X., and Marteau, P. (2008). A double blind randomized controlled trial of a probiotic combination in 100 patients with irritable bowel syndrome. Gastroenterol. Clin. Biol. 32, 147-152. doi: 10.1016/j.gcb.2007.06.001

Ethan, G., Henna, S., and Amee, R. M. (2011). Systematic review of intestinal microbiota transplantation (fecal bacteriotherapy) for recurrent clostridium difficile infection. Clin. Infect. Dis 53, 994-1002. doi: 10.1093/cid/c ir632

Everard, A., Belzer, C., Geurts, L., Ouwerkerk, J. P., and Cani, P. D. (2013). Cross-talk between Akkermansia muciniphila and intestinal epithelium controls diet-induced obesity. Proc. Natl. Acad. Sci. U.S.A. 110, 9066-9071. doi: 10.1073/ pnas. 1219451110

Faith, D. P., and Baker, A. M. (2006). Phylogenetic diversity (PD) and biodiversity conservation: some bioinformatics challenges. Evol. Bioinform. Online 2, 121128. doi: $10.1177 / 117693430600200007$

Ge, X., Zhao, W., Ding, C., Tian, H., Xu, L., and Wang, H. (2017). Potential role of fecal microbiota from patients with slow transit constipation in the regulation of gastrointestinal motility. Sci. Rep. 7:441. doi: 10.1038/s41598-01700612-y

Guérin, A., Mody, R., Fok, B., Lasch, K. L., Zhou, Z., Wu, E. Q., et al. (2014). Risk of developing colorectal cancer and benign colorectal neoplasm in patients with chronic constipation. Aliment. Pharmacol. Ther. 40, 83-92. doi: 10.1111/apt. 12789

Guilloteau, P., Martin, L., Eeckhaut, V., Ducatelle, R., Zabielski, R., and Van Immerseel, F. (2010). From the gut to the peripheral tissues: the multiple effects of butyrate. Nutr. Res. Rev. 23, 366-384. doi: 10.1017/S0954422410000247

Jenq, R. R., Ubeda, C., Taur, Y., Menezes, C. C., Khanin, R., and Dudakov, J. A. (2012). Regulation of intestinal inflammation by microbiota following allogeneic bone marrow transplantation. J. Exp. Med. 209, 903-911. doi: 10. 1084/jem.20112408

Konikoff, T., and Gophna, U. (2016). Oscillospira: a central, enigmatic component of the human gut microbiota. Trends Microbiol. 24, 523-524. doi: 10.1016/j.tim. 2016.02.015

Ley, R. E. (2016). Prevotella in the gut: choose carefully. Nat. Rev. Gastroenterol. Hepatol. 13, 69-70. doi: 10.1038/nrgastro.2016.4

Mao, B., Li, D., Zhao, J., Ai, C., Zhang, H., and Chen, W. (2016). Lactulose differently modulates the composition of luminal and mucosal microbiota in C57BL/6J Mice. J. Agri. Food Chem. 64, 6240-6247. doi: 10.1021/acs.jafc. 6b02305

Michael, C. A., Peter, T. M., and Eric, G. P. (2016). Clostridium difficile colitis: pathogenesis and host defence. Nat. Rev. Microbiol. 14, 609-620. doi: 10.1038/ nrmicro.2016.108

Ormerod, K. L., Wood, D. L. A., Lachner, N., Gellatly, S. L., Daly, J. N., and Parsons, J. D. (2016). Genomic characterization of the uncultured bacteroidales familys 24-7 inhabiting the guts of homeothermic animals. Microbiome. 4:36. doi: 10.1186/s40168-016-0181-2

Parthasarathy, G., Chen, J., Chen, X., Chia, N., O'Connor, H. M., Wolf, P. G., et al. (2016). Relationship between microbiota of the colonic mucosa vs feces and symptoms, colonic transit, and methane production in female patients with chronic constipation. Gastroenterology 150, 367-379.e1. doi: 10.1053/j.gastro. 2015.10.005

Peter, J. W., Linda, A., Morel, J., Bond, Y., Charbonneau, D., and O’Mahony, L. (2006). Efficacy of an encapsulated probiotic bifidobacterium infantis 35624 in women with irritable bowel syndrome. Am. J. Gastroenterol. 101, 1581-1590. doi: 10.1111/j.1572-0241.2006.00734.- X

Qian, Y., Zhao, X., and Kan, J. (2013). Preventive effect of resistant starch on activated carbon-induced constipation in mice. Exp. Ther. Med. 6, 228-232. doi: $10.3892 /$ etm.2013.1096
Rao, S. S., Rattanakovit, K., and Patcharatrakul, T. (2016). Diagnosis and management of chronic constipation in adults. Nat. Rev. Gastroenterol. Hepatol. 13, 295-305. doi: 10.1038/nrgastro.2016.53

Reigstad, C. S., Salmonson, C. E., Rainey, J. F., Szurszewski, J. H., Linden, D. R., Sonnenburg, J. L., et al. (2015). Gut microbes promote colonic serotonin production through an effect of short-chain fatty acids on enterochromaffin. FASEB J. 29, 1395-1403. doi: 10.1096/fj.14-259598/fj.14259598

Ríos-Covián, D., Patricia, R. M., Abelardo, M., Gueimonde, M., de Los ReyesGavilán, C. G., and Salazar, N. (2016). Intestinal short chain fatty acids and their link with diet and human health. Front. Microbiol. 7:185. doi: 10.3389/fmicb. 2016.00185

Rooks, M. G., Veiga, P., Wardwell-Scott, L. H., Tickle, T., Segata, N., and Michaud, M. (2014). Gut microbiome composition and function in experimental colitis during active disease and treatment-induced remission. ISME J. 8, 1403-1417. doi: 10.1038 /ismej.2014.3

Rossi, L., Marcq, E., Montmessin, F., Fedorova, A., Stam, D., Bertaux, J.-L., et al. (2015). Preliminary study of Venus cloud layers with polarimetric data from SPICAV/VEx. Planet. Space Sci. 113-114, 159-168. doi: 10.1016/j.pss.2014.11. 011

Sadik, R., Abrahamsson, H., Ung, K. A., and Stotzer, P. O. (2004). Accelerated regional bowel transit and overweight shown in idiopathic bile acid malabsorption. Gastroenterol. 124, A145-A145.

Salminen, S., and Salminen, E. (1997). Lactulose, lactic acid bacteria, intestinal microecology and mucosal protection. Scand. J. Gastroenterol. Suppl. 222, 45-48. doi: 10.1080/00365521.1997.11720717

Seyed, S. M., Seyed, A. M., Nabavizadeh, B., Entezari, P., Ostovaneh, M. R., and Hosseini, S. M. E. (2015). Impact of microscopic duodenitis on symptomatic response to Helicobacter pylori eradication in functional dyspepsia. Digest. Dis. Sci. 60, 163-167. doi: 10.1007/s10620-0143285-1

Sokol, H., Pigneur, B., Watterlot, L., Lakhdari, O., Bermudez-Humaran, L. G., and Gratadoux, J. J. (2008). Faecalibacterium prausnitzii is an anti-inflammatory commensal bacterium identified by gut microbiota analysis of crohn disease patients. Proc. Natl. Acad. Sci. U.S.A. 105, 16731-16736. doi: 10.1073/pnas. 0804812105

Sostres, C., Carrera, L. P., Benito, R., Roncales, P., Arruebo, M., and Arroyo, M. T. (2015). Peptic ulcer bleeding risk. the role of helicobacter pylori infection in nsaid/low-dose aspirin users. Am. J. Gastroenterol. 110, 684-689. doi: 10.1038/ ajg. 2015.98

Tabbers, M. M., Milliano, I. D., Roseboom, M. G., and Benninga, M. A. (2011). Is bifidobacterium breve effective in the treatment of childhood constipation? results from a pilot study. Nutr. J. 10:19. doi: 10.1186/1475-289110-19

Urita, Y., Goto, M., Toshiyasu, W., Makoto, M., Atsushi, G., and Mitsuyoshi, K. (2015). Continuous consumption of fermented milk containing bifidobacterium bifidum yit 10347 improves gastrointestinal and psychological symptoms in patients with functional gastrointestinal disorders. Biosci. Microbiota Food Health 34, 37-44. doi: 10.12938/bmfh. 2014-017

Veiga, P., Pons, N., Agrawal, A., Oozeer, R., and Kennedy, S. P. (2014). Changes of the human gut microbiome induced by a fermented milk product. Sci Rep. 4, 6328. doi: 10.1038/srep06328

Vidyasagar, S., and Ramakrishna, B. S. (2000). Effects of butyrate on active sodium and chloride transport in rat and rabbit distal colon. J. Physiol. 539, 163-173. doi: 10.1113/jphysiol.2001.013056

Wang, L. L., Hu, L. J., Xu, Q., Yin, B. X., Fang, S. G., Wang, G., et al. (2017a). Bifidobacterium adolescentis exerts strain-specific effects on constipation induced by loperamide in balb/c mice. Int. J. Mol. Sci. 18:318. doi: 10.3390/ ijms 18020318

Wang, L. L., Hu, L. J., Yan, S., Jiang, T., Fang, S. G., Wang, G., et al. (2017b). Bifidobacteria exerts species-specific effects on constipation in BALB/c mice. Food Funct. 8, 3587-3600. doi: 10.1039/C6FO01641C

Watari, J., Chen, N., Peter, S. A., Hirokazu, F., Tadayuki, O., Toshihiko, T., et al. (2014). Helicobacter pylori associated chronic gastritis, clinical syndromes, precancerous lesions, and pathogenesis of gastric cancer development. World J. Gastroenterol. 20, 5461-5473. doi: 10.3748/wjg.v20.i18. 5461 
Williams, E. A., Stimpson, J., Wang, D., Plummer, S., Garaiova, I., Barker, M. E., et al. (2009). Clinical trial: a multistrain probiotic preparation significantly reduces symptoms of irritable bowel syndrome in a double-blind placebocontrolled study. Aliment. Pharmacol. Ther. 29, 97-103. doi: 10.1111/j.13652036.2008.03848.x

Yajima, T. (1988). Luminal propionate-induced secretory response in the rat distal colon in vitro. J. Physiol. 403, 559-575. doi: 10.1113/jphysiol.1988.sp017264

Yoon, H., Park, Y. S., Lee, D. H., Seo, J. G., Shin, C. M., and Kim, N. A. (2015). Effect of administering a multi-species probiotic mixture on the changes in fecal microbiota and symptoms of irritable bowel syndrome: a randomized, double-blind, placebo-controlled trial. J. Clin. Biochem. Nutr. 57, 129-134. doi: $10.3164 /$ jcbn. 1514

Zhang, J., Guo, Z., Xue, Z., Sun, Z., Zhang, M., and Wang, L. (2015). A phylofunctional core of enteric microorganisms in healthy young chinese cohorts across lifestyles, geography and ethnicities. ISME J. 9, 1979-1990. doi: 10.1038/ ismej.2015.11

Conflict of Interest Statement: The authors declare that the research was conducted in the absence of any commercial or financial relationships that could be construed as a potential conflict of interest.

Copyright $\odot 2019$ Wang, Chen, Cui, Lee, Wang, Zhao, Zhang and Chen. This is an open-access article distributed under the terms of the Creative Commons Attribution License (CC BY). The use, distribution or reproduction in other forums is permitted, provided the original author(s) and the copyright owner(s) are credited and that the original publication in this journal is cited, in accordance with accepted academic practice. No use, distribution or reproduction is permitted which does not comply with these terms. 